Indonesian Journal of Physics

Vol 20 No. 1, January 2009

\title{
Anneal Temperature Effect on Crystallite Size and Electric Conductivity of $\mathrm{LiMn}_{2} \mathrm{O}_{4}$
}

\author{
Khairul Basar $^{a)}$, Xianglian $^{\text {b) }}$, Sainer Siagian ${ }^{\text {) }}$, Kouta Ohara $^{\text {b) }}$, Takashi Sakuma ${ }^{\text {() }}$, \\ Haruyuki Takahashi ${ }^{\text {b) }}$, Osami Abe ${ }^{\text {c) }}$, Naoki Igawa ${ }^{d)}$ and Yoshinobu Ishii ${ }^{d)}$ \\ a) Faculty of Mathematics and Natural Sciences, \\ Institut Teknologi Bandung, Bandung 40132, Indonesia \\ b) Institute of Applied Beam Science, Ibaraki University, \\ Hitachi 316-8511, Japan \\ c) Faculty of Engineering, Ibaraki University, \\ Hitachi 316-8511, Japan \\ d) Quantum Beam Science Directorate, \\ Japan Atomic Energy Agency, Tokai 319-1195, Japan \\ e-mail:khbasar@gmail.com
}

\begin{abstract}
Powder samples of $\mathrm{LiMn}_{2} \mathrm{O}_{4}$ have been prepared by solid state reaction of $\mathrm{LiOH}$ and $\mathrm{MnO}_{2}$. Neutron scattering experiments at room temperature and conductivity measurement have been performed on the samples. Anneal temperature effect on the crystallite size and electric conductivity of $\mathrm{LiMn}_{2} \mathrm{O}_{4}$ is analyzed. The average crystallite size is obtained from the full width at half maximum (FWHM) of Bragg lines using Scherrer equation. The average crystallite size increases with the increase of anneal temperature. Activation energy and conductivity prefactor increase with anneal temperature.
\end{abstract}

Keywords: Neutron scattering, $\mathrm{LiMn}_{2} \mathrm{O}_{4}$, Crystallite size, Electric conductivity

\section{Introduction}

Lithium-based metal oxides are well known promising materials for cathode in next generation of rechargeable lithium-ion batteries due to their high energy densities. At present, $\mathrm{LiCoO}_{2}$ is widely used as commercial product for cathode in lithium-ion batteries. However the cobalt in $\mathrm{LiCoO}_{2}$ is a relatively rare metal, expensive and toxic. Nowadays, $\mathrm{LiNiO}_{2}$ and $\mathrm{LiMn}_{2} \mathrm{O}_{4}$ are studied as alternative materials because they are relatively cheaper and less toxic. $\mathrm{LiMn}_{2} \mathrm{O}_{4}$ is electrochemically more stable than $\mathrm{LiNiO}_{2}$.

In recent years, there is a great interest in the synthesis of nanosized materials for development of cathode in lithium-ion batteries ${ }^{1,2}$. It has been reported that anneal process is important to control the crystal size in the lithium-based metal oxide ${ }^{3)}$. It is known that charge-discharge characteristics of lithium cells are mainly depend on the physical and chemical properties of the cathode materials such as crystallite size, purity and homogeneity ${ }^{4,5)}$. Effect of annealing on the charge-discharge properties of $\mathrm{LiMn}_{2} \mathrm{O}_{4}$ has been reported ${ }^{6)}$. The synthesis process is important to obtain the materials with desired properties. From the point of possible application of $\mathrm{LiMn}_{2} \mathrm{O}_{4}$ in lithium ion batteries, it is important to understand the formation process of crystal $\mathrm{LiMn}_{2} \mathrm{O}_{4}$ by anneal treatment and its relation to the electrical properties. In this paper we report the anneal temperature effect on crystallite size of $\mathrm{LiMn}_{2} \mathrm{O}_{4}$ and its relation to the electric conductivity.

\section{Experimental}

Powder sample of $\mathrm{LiMn}_{2} \mathrm{O}_{4}$ was from starting materials $\mathrm{LiOH}$ and $\mathrm{MnO}_{2}$. The mixture was milled by high-energy planetary milling for 3 hours at frequency $2 \mathrm{~Hz}$ to perform a ground product. After that, it was heated at temperature $200^{\circ} \mathrm{C}$ for 16 hours. In order to analyze the anneal temperature effect to the crystallite size and electric conductivity of $\mathrm{LiMn}_{2} \mathrm{O}_{4}$, the whole sample was divided into three parts: sample A which was annealed at $400^{\circ} \mathrm{C}$ for 3 hours, sample B annealed at $500^{\circ} \mathrm{C}$ for 3 hours and sample $\mathrm{C}$ annealed at $800^{\circ} \mathrm{C}$ for 3 hours.

Neutron scattering experiments have been performed on $\mathrm{LiMn}_{2} \mathrm{O}_{4}$ (sample A, B and C) at room temperature using HRPD (High Resolution Powder Diffractometer) installed at Japan Research Reactor 3 in Japan Atomic Energy Agency (JAEA). The experimental set-up was including a $12^{\prime}$ collimator in front of monochromator. Incident neutron wavelength of $1.823 \AA$ which was monochromatized by Ge (331) was used and the data were collected in the $2 \theta$ range from $10^{\circ}$ to $150^{\circ}$ with step angle $0.05^{\circ}$.

Powder samples for conductivity measurements were pressed between conductive silver powder layer at $700 \mathrm{~kg} / \mathrm{cm}^{2}$ for about 1 hour into cylindrical pellets of $1.3 \mathrm{~cm}$ in diameter and $\sim 1 \mathrm{~mm}$ in thick. The entire cell was clamped with nonconductive plate and inserted into a special vacuum vessel. The measurements were done using KOKUYO KC-605 LCR Meter at temperature range from room temperature to $100^{\circ} \mathrm{C}$.

\section{Results and Discussion}

Figure 1 shows neutron scattering intensity from sample A, B and C after experimental time correction. Neutron scattering intensity of sample A shows very weak and wide Bragg peaks of $\mathrm{LiMn}_{2} \mathrm{O}_{4}$ around $2 \theta \sim 45^{\circ}, 95^{\circ}$ and $118^{\circ}$. These three peaks 
become stronger and narrower in the sample $\mathrm{B}$. Other clear Bragg peaks of $\mathrm{LiMn}_{2} \mathrm{O}_{4}$ also appear at $52.5^{\circ}$, $57.5^{\circ}, 70^{\circ}$ and $77^{\circ}$ in the scattering intensity of sample B. The strongest and narrowest Bragg peaks is observed in the sample C.

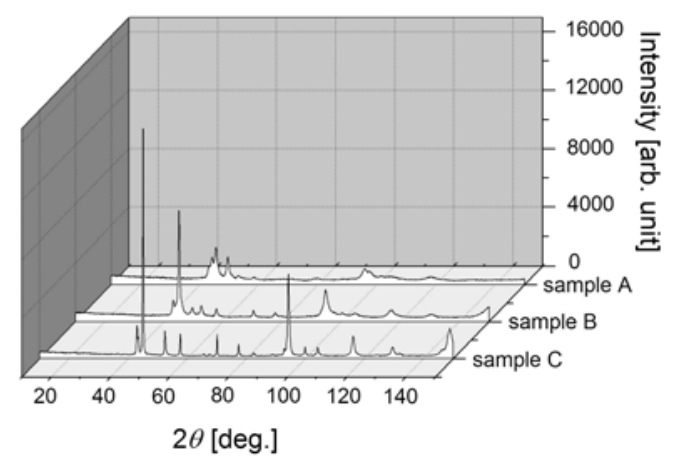

Figure 1. Neutron scattering intensities of powder $\mathrm{LiMn}_{2} \mathrm{O}_{4}$ at room temperature by HRPD.

In the former report of another lithium-based metal oxide $\mathrm{LiNiO}_{2}$, the diffuse scattering intensity decreases drastically with increasing the annealing temperature. The strong diffuse neutron scattering intensity is related to the amount of starting material $\mathrm{LiOH}$ in the powder samples, because atom $\mathrm{H}$ has large incoherent scattering cross section by neutron beam ${ }^{3)}$. However, in the case of $\mathrm{LiMn}_{2} \mathrm{O}_{4}$ the diffuse scattering intensity does not change so much in sample A, B and C as shown in Fig. 1. It is concluded that the amount of starting material $\mathrm{LiOH}$ is very small even in sample A. Furthermore, this means that the solid state reaction to perform $\mathrm{LiMn}_{2} \mathrm{O}_{4}$ is almost completed by annealing the sample at $400^{\circ} \mathrm{C}$.

Rietveld refinement analysis has been performed on the neutron scattering intensities of sample A, B and C using RIETAN-20007). Crystal of $\mathrm{LiMn}_{2} \mathrm{O}_{4}$ is assumed to belong to cubic type structure with the space group $F d \overline{3} m^{8}$. It is obtained that our structure model fitted well with the experimental data. In Table 1, $R$ factors and structure parameters obtained from refinement analysis are shown.

Table 1. Reliability factors $R$, goodness of fit parameter $S$, lattice constant $a$, coordinate fraction of atom $\mathrm{O} x$, and temperature parameters $B$ obtained from refinement analysis.

\begin{tabular}{c|c|c|c|c|c|c|c|c|c}
\hline sample & $\begin{array}{c}R_{\mathrm{wp}} \\
{[\%]}\end{array}$ & $\begin{array}{c}R_{\mathrm{I}} \\
{[\%]}\end{array}$ & $\begin{array}{c}R_{\mathrm{F}} \\
{[\%]}\end{array}$ & $S$ & $\begin{array}{c}a \\
{[\AA]}\end{array}$ & $x$ & $\begin{array}{c}B_{\mathrm{Li}} \\
{\left[\AA^{2}\right]}\end{array}$ & $\begin{array}{c}B_{\mathrm{Mn}} \\
{\left[\AA^{2}\right]}\end{array}$ & $\begin{array}{c}B_{\mathrm{O}} \\
{\left[\AA^{2}\right]}\end{array}$ \\
\hline $\mathrm{A}$ & 8.87 & 5.34 & 2.19 & 1.91 & 8.234 & 0.3875 & 0.110 & 0.520 & 0.980 \\
$\mathrm{~B}$ & 8.01 & 4.16 & 3.48 & 1.82 & 8.236 & 0.3880 & 0.119 & 0.644 & 1.079 \\
$\mathrm{C}$ & 11.42 & 5.60 & 4.17 & 2.27 & 8.236 & 0.3880 & 0.458 & 0.723 & 1.161 \\
\hline
\end{tabular}

Table 2. Values of instrumental correction $D_{\text {inst }}$, full width at half maximum (FWHM) from refinement analysis $D_{\text {meas }}$ and corrected FWHM $D$ of Bragg peaks in sample A, B and C which were used in the calculation of Scherrer equation.

\begin{tabular}{c|c|c|c|c|c|c|c|c}
\hline & $2 \theta$ & $\begin{array}{c}D_{\text {inst }} \\
\text { [deg.] }\end{array}$ & \multicolumn{3}{|c|}{$\begin{array}{c}D_{\text {meas }} \\
\text { [deg.] }\end{array}$} & \multicolumn{3}{|c}{$D=\left(D_{\text {meas }}^{2}-D_{\text {inst }}^{2}\right)^{1 / 2}$} \\
[deg.] \\
\end{tabular}

The average crystallite size of $\mathrm{LiMn}_{2} \mathrm{O}_{4}$ formed in the annealing process from ground product is analyzed from the full width at half maximum of Bragg line using Scherrer equation:

$$
t=\frac{K \lambda}{D \cos \theta},
$$

where $t$ is the average crystallite size in angstrom, $K$ is a constant and equal to $0.9, \lambda$ the wavelength of incident neutron beam, $D$ the FWHM (full width at half maximum) of Bragg line and $2 \theta$ the scattering angle of Bragg line. For each value of $h k l$-reflection, the value of $t$ is interpreted as an average crystal dimension perpendicular to the reflecting planes. The full width at half maximum of Bragg line $D_{\text {meas }}$ which is obtained from measurements should be corrected with the instrumental resolution $D_{\text {inst }}$. The corrected FWHM $D$ is obtained by using the relation:

$$
D^{2}=D_{\text {meas }}^{2}-D_{\text {inst }}^{2} \text {. }
$$

The value of $D_{\text {meas }}$ was obtained from Rietveld refinement analysis.

The crystallite size of $\mathrm{LiMn}_{2} \mathrm{O}_{4}$ in samples A, $\mathrm{B}$ and $\mathrm{C}$ are calculated using three Bragg peaks (400), (331) and (622). The values of FWHM from refinement $D_{\text {meas }}$ for these three Bragg peaks and 
instrumental resolution $D_{\text {inst }}$ at $2 \theta$ of the peaks are shown in Table 2. The average crystallite sizes of $\mathrm{LiMn}_{2} \mathrm{O}_{4}$ are $~ 5,14$ and $37 \mathrm{~nm}$ for sample A, B and C, respectively as shown in Fig. 2. From the analysis of crystallite size using Scherrer equation, it is shown that the average size increases almost linearly with annealing temperature.

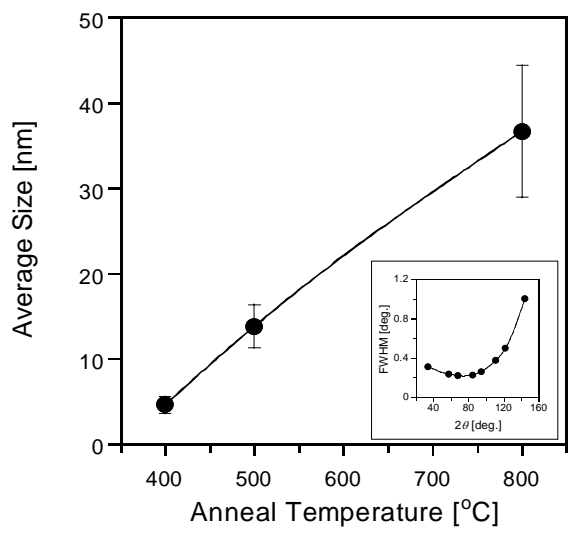

Figure 2.Average crystallite size of $\mathrm{LiMn}_{2} \mathrm{O}_{4}$ in powder samples. Inset shows the resolution curve of the instrument.

Figure 3 shows the DC conductivity $\sigma_{\mathrm{DC}}$ of sample A, B and C. The temperature dependence of $\sigma_{\mathrm{DC}}$ is analyzed using Arrhenius relation:

$$
\sigma_{\text {DC }}=\sigma_{\mathrm{o}} \exp \left(-\frac{E_{\mathrm{a}}}{k T}\right),
$$

where $\sigma_{0}$ is conductivity prefactor, $E_{\text {a }}$ activation energy, $k$ Boltzmann constant and $T$ temperature in Kelvin. DC conductivity shows clearly temperature dependence and obeys Arrhenius relation. Our results on DC conductivity agree with the previous report ${ }^{12)}$. Anneal temperature also affects to the value of DC conductivity. DC conductivity of sample B at one specific temperature is higher than that of sample $A$ and $\mathrm{C}$ as shown in Fig. 3(a). In Fig. 3(b) the DC conductivities of sample $\mathrm{A}, \mathrm{B}$ and $\mathrm{C}$ at room temperature are shown. In addition to the DC conductivity data of sample A, B and C, the DC conductivities at room temperature for samples which were annealed at 700 and $900^{\circ} \mathrm{C}$ are also checked. The values of DC conductivity of these two additional samples are marked with (\#) in Fig. 3(b). Solid line in Fig. 3(b) is figured for the reader's eye to show the tendency of DC conductivity. Based on the anneal temperature dependence of DC conductivity data, it might be possible to suggest that there is an optimum annealing temperature between 500 and $800^{\circ} \mathrm{C}$ to obtain high DC conductivity. This might be related to the crystallite size and grain boundary resistivity. However, further analysis is needed to confirm this.
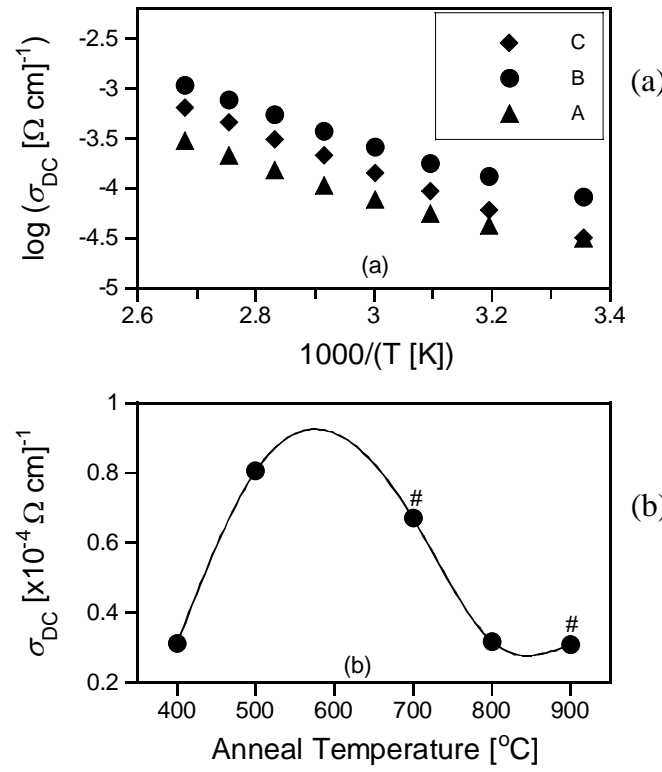

(b)

Figure 3. (a) Arrhenius plot of conductivity of $\mathrm{LiMn}_{2} \mathrm{O}_{4}$ samples, (b) $\mathrm{DC}$ conductivity of $\mathrm{LiMn}_{2} \mathrm{O}_{4}$ samples at room temperature.

The conductivity prefactor $\sigma_{0}$ and activation energy $E_{\mathrm{a}}$ are obtained by linear least squares fitting to the observed DC conductivity data using eq. (3) and its values are shown in Fig. 4. A relatively small variation of activation energy between $0.30-0.39 \mathrm{eV}$ were obtained for sample A, B and C. It is obtained that the values of conductivity prefactor and activation energy increase with increasing anneal temperature.

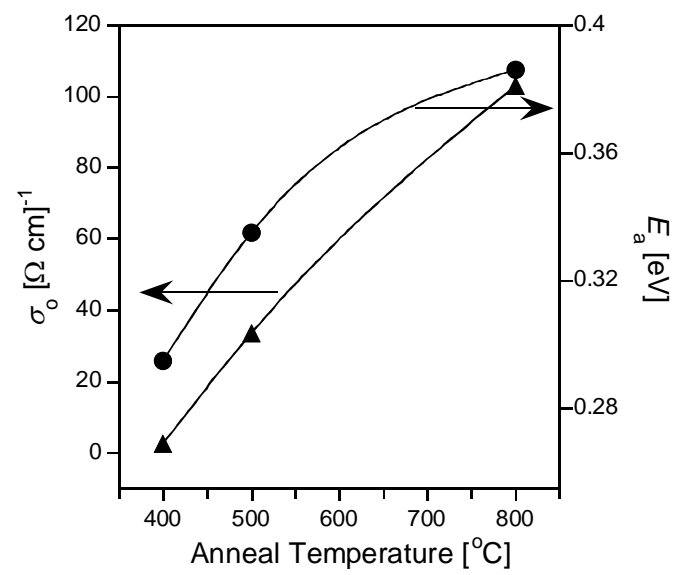

Figure 4. Conductivity prefactor $\sigma_{0}$ and activation energy $E_{\mathrm{a}}$ of $\mathrm{LiMn}_{2} \mathrm{O}_{4}$. 


\section{Conclusions}

Nanosized $\mathrm{LiMn}_{2} \mathrm{O}_{4}$ has been successfully prepared by solid state reaction of $\mathrm{LiOH}$ and $\mathrm{MnO}_{2}$. The average crystallite sizes of $\mathrm{LiMn}_{2} \mathrm{O}_{4}$ in powder samples increases with increasing anneal temperature. From the conductivity measurements, it was concluded that there is an optimum anneal temperature to obtain high DC conductivity at room temperature from powder $\mathrm{LiMn}_{2} \mathrm{O}_{4}$.

\section{Acknowledgements}

Financial supports from The Ministry of Education, Science, Sports and Culture, Grant-in-Aid for Scientific Research on Priority Area, 17041001, 2006 and from Ibaraki Prefecture are gratefully acknowledged. One of the authors (KB) expresses his thanks to Ibaraki Prefecture, Hitachi, Ltd. and Ibaraki University for research fellowship by Renkei Jigyou.

\section{References}

1. S. H. Ye et al., Electrochimica Acta, 49, 1623, 2004.

2. T. Savitha, S. Selvasekarapandian, and C. S. Ramya, in: B. V. R. Chowdari et al,. (Eds.), Solid
State Ionics, World Scientific Pub., Singapore, 2006, p. 337.

3. K. Basar et al., in: B. V. R. Chowdari et al., (Eds.), Solid State Ionics, World Scientific Pub., Singapore, 2006, p. 121.

4. C. Tsang and A. Manthiram, Solid State Ionics, 89, 305, 1996.

5. T. Tsumura, A. Shimizu, and M. Inagaki, Solid State Ionics, 90, 197, 1996.

6. F. -Y. Shih and K. -Z. Fung, J. Alloy and Compounds, 430, , 320, 2007.

7. F. Izumi and T. Ikeda, Mater. Sci. Forum, 321324, 198, 2000.

8. K. S. Yoo, N. W. Cho, and Y. -J. Oh, Solid State Ionics, 113-115, 43, 1998.

9. D. P. Almond, C. C. Hunter, and A. R. West, J. Mater. Sci. 19, 3236, 1984.

10. C. Cramer and M. Buscher, Solid State Ionics, 105, 109, 1998

11. K. Funke and D. Wilmer, Solid State Ionics, 136137, 1329, 2000.

12. J. Marzec et al., Solid State Ionics, 146, 225, 2002. 